\title{
Cuidadores familiares principales de niños con cáncer y apoyo social recibido, Cartagena
}

\author{
Primary family caregivers of children with cancer and their available social support in Cartagena (Colombia)
}

Amparo Astrid Montalvo-Prieto1* orcid.org/0000-0002-7925-8674

Yenifer Tatiana Peluffo-Mendoza' orcid.org/0000-0001-9063-6333

1. Programa Enfermería, Universidad de Cartagena. Cartagena, Colombia.

Fecha de recepción: Junio 19 - $2019 \quad$ Fecha de revisión: Septiembre 23 - $2019 \quad$ Fecha de aceptación: Diciembre 29 - 2020

Montalvo-Prieto AA. Peluffo Mendoza YT. Cuidadores familiares principales de niños con cáncer y apoyo social recibido. Cartagena. Univ. Salud. 2021;23(1):13-20. DOI: https://doi.org/10.22267/rus.212301.209

\section{Resumen}

Introducción: El apoyo social para los cuidadores de los niños con cáncer, es una fuente de ayuda que los fortalece para enfrentar esta situación. Objetivo: Determinar la asociación entre las condiciones sociodemográficas y de relación de cuidado, con el apoyo social total percibido y requerido por los cuidadores familiares principales de niños con cáncer, en dos instituciones de salud de Cartagena-Colombia. Materiales y métodos: Estudio transversal analítico con 126 cuidadores. Se utilizaron los instrumentos de caracterización de cuidadores familiares de personas con enfermedad crónica ${ }^{\circledR}$ y Duke-UNK-11. Los resultados se presentan en valores absolutos y relativos, y las asociaciones con Odds ratio. Resultados: El 42,9\% son únicos cuidadores, con edades entre 18 a 59 años, 78,8\% son mujeres. El 56,3\% ha sido cuidador por más de 19 meses, 94,4\% desde el diagnóstico, 50\% cuida todo el día. El 38,9\% considera escaso el apoyo social, 75,4\% el apoyo confidencial y $23 \%$ el afectivo. Conclusiones: Ser cuidador ocasiona soledad, cansancio, abandono de su propio cuidado y el apoyo emocional se convierte en un recurso para fortalecerlo; sin embargo, los cuidadores del presente estudio no lo perciben. Se requiere liderazgo para desarrollar formación en valores de convivencia, para visibilizar el apoyo social en cuidadores.

Palabras clave: Apoyo social; cuidador familiar; cáncer; niños. (Fuente: DeCS, Bireme).

\begin{abstract}
Introduction: Social support for caregivers of children with cancer is a source of help that strengthens them to face this situation. Objective: To determine the association between sociodemographic and care conditions with the perceived and actual social support received by primary family caregivers of children with cancer in two health institutions in Cartagena-Colombia. Materials and methods: Cross-sectional analytical study with 126 caregivers, using cronica(C) and Duke-UNK-11 instruments to characterize family caregivers of people with chronic diseases. The results are presented in absolute and relative values and the associations are shown as Odds ratio. Results: 42.9\% of caregivers function alone, with ages between 18 and 59 years. $78.8 \%$ are women and $56.3 \%$ have been caregivers for more than 19 months, of which $94.4 \%$ have been caregivers since the diagnosis. $50 \%$ serve as caregivers all day, $38.9 \%$ report that social support is scarce, $75.4 \%$ that support is confidential and $23 \%$ report it as affective. Conclusions: Being a caregiver causes loneliness, fatigue and neglect of their own care. Even though emotional support of caregivers becomes an important resource to strengthen them, the participating caregivers did not perceive it. Leadership is required to foster values of coexistence and make social support available for caregivers.
\end{abstract}

Keywords: Social support; Caregivers; cancer; child. (Fuente: DeCS, Bireme).

\footnotetext{
*Autor de correspondencia

Amparo Astrid Montalvo Prieto

e-mail: amontalvop1@unicartagena.edu.co
} 


\section{Introducción}

El cáncer es una enfermedad de alta incidencia en niños, la Organización Mundial de la Salud (OMS) designa diferentes tipos de patologías que pueden aparecer antes de cumplir los 15 años(1), cerca de 300.000 niños en el mundo reciben diagnóstico de cáncer al año, de los cuales la tasa de curación en los países de medianos y bajos ingresos es de aproximadamente el $20 \%$ de los niños y del $80 \%$ para los países de ingresos altos(2). En Colombia el cáncer es la segunda causa de muerte infantil en el país y el tipo más común es la leucemia. En el año 2016 se registraron 1.200 casos, muchos de ellos fallecieron en el primer año(3). En Cartagena, la incidencia de cáncer en menores de 18 años es de 13,7 casos por 100.000 y una tasa de mortalidad de 3,2 casos por 100.000 (4).

La detección del cáncer en uno de los miembros de la familia, conlleva un cambio radical en el interior del individuo y su entorno, este diagnóstico significa una crisis para la familia y un cambio en su estilo de vida(5). Ante esta situación un miembro de la familia asume de manera implícita o explícita las funciones como cuidador(6), que aunque no pertenece al equipo de salud institucional, ni ha recibido formación, toma la responsabilidad del cuidado y atención en casa del paciente(7).

Pinzón y Carrillo(8) anotan que los cuidadores familiares lo son desde el inicio de la enfermedad y esta circunstancia puede deberse a que viven en la misma casa de su familiar enfermo, esto los induce a asumir el compromiso y las labores del cuidado. Ante un niño enfermo con cáncer, es la madre quien generalmente toma el compromiso como principal cuidador, aunque Fernández et al.,(7) reportan también al padre como cuidador en un porcentaje significativo.

La carga física y emocional a la que están sometidos los cuidadores familiares es alta, en la que además de padecer la incertidumbre acerca de la evolución de la enfermedad en su niño, está presente la gran carga económica, la vulnerabilidad y su dependencia. Estas circunstancias le ocasionan al cuidador diversas manifestaciones emocionales como sufrimiento $\mathrm{y}$ frustración(7). Pozo et al.(9) en su estudio describen los malestares que sufren los padres por las visitas al hospital, los exámenes médicos y el tratamiento que les administran.
Esta situaciones con frecuencia provocan en el cuidador sobrecargas, preocupaciones y estrés, porque además de la responsabilidad de proporcionar cuidado, deben participar en la toma de decisiones, identificar las necesidades del paciente, verificar el desarrollo de acciones cotidianas y estar atento a acompañar al paciente en las visitas a los centros asistenciales, realización de exámenes, motivarlo y apoyarlo emocionalmente, a fin de contribuir al mejoramiento de su calidad de vida(7). Estas situaciones estresantes pueden ser manejadas de forma positiva por el cuidador familiar de acuerdo a su edad, la escolaridad y el nivel de alteración cognitiva que presenta(10). De manera complementaria Achury et al.(11) plantean que la confianza que tienen los cuidadores en su capacidad para asumir su rol, mantener una buena comunicación y vínculo afectivo con la persona que se cuida son importantes en la relación de cuidado(11).

El apoyo social efectivo es fundamental para la actividad de cuidado y está influenciado por el número de personas que acompañan al cuidador en esta actividad(12). Para los cuidadores, el apoyo que reciben de la familia es muy importante, puesto que les permite enfrentar de una manera más efectiva las situaciones estresantes que le generan su rol de cuidador(7).

De acuerdo a lo afirmado por Ostertag(13), el apoyo social positivo y efectivo, está relacionado con los procesos de adaptación y afrontamiento que desarrolle el cuidador familiar. En su estudio Carrillo et al.,(14) informan que los cuidadores de niños con cáncer presentaron poca habilidad de cuidado para afrontar su rol, traducida ésta en las habilidades de valor, paciencia y conocimiento, ocasionando que un número limitado de cuidadores presenten un nivel de responsabilidad adecuado para afrontar el rol de cuidador(14).

Para los niños, el cuidador familiar principal se constituye en la fuente fundamental de cuidado, pero es necesario que este cuente con una red social que le ayude en el afrontamiento del diagnóstico, el tratamiento y acompañamiento. Varios cuidadores se sienten solos en su labor, consideran que los médicos y enfermeras que atendieron a su niño se convierten en apoyo durante la enfermedad, informan que algunas fundaciones les brindan ayuda a ellos y a los niños que cuidan y señalan que las 
prácticas religiosas les proporcionan fortalecimiento espiritual (12-14).

Para enfermería el conocimiento acerca de la importancia, reconocimiento y estímulo del apoyo social a los cuidadores, se convierte en una información significativa, porque permite desde la formación académica, identificar sus características, el tipo de apoyo que se brinda, de tal forma que se desarrollen planes que permita su fortalecimiento. El propósito del presente estudio fue determinar la asociación entre las condiciones sociodemográficas y de relación de cuidado con el apoyo social total percibido y requerido por los cuidadores familiares principales de niños con cáncer en dos instituciones de salud de Cartagena, Colombia.

\section{Materiales y métodos}

Estudio trasversal de tipo analítico, realizado durante los años 2016-2017 en dos instituciones de salud de Cartagena (Fundevida y Hospital infantil Franco Pareja). La población de estudio fueron ciento veintiséis (126) cuidadores familiares principales de niños con cáncer, que cumplieron criterios de inclusión de ser mayores de 18 años de edad, con niños de más de un año de evolución de la enfermedad y participar voluntariamente en la investigación.

Se utilizaron dos instrumentos de evaluación: la encuesta de caracterización de cuidadores familiares de personas con enfermedad crónica $($, con un alfa de Cronbach de 0,683(15), que consta de 14 ítems que contempla aspectos sociodemográficos y de relación de cuidado, su uso fue autorizado por los autores y el cuestionario de apoyo social funcional DUKE-UNC11 validado al español, con un Alfa de Cronbach de 0,92(16), que tiene de 11 ítems, evalúa dos dimensiones, apoyo confidencial y afectivo, en una escala tipo Likert con cinco posibles respuestas (1 a $5)$, donde 1 corresponde a mucho menos de lo que deseo, 2 menos de lo que deseo, 3 ni mucho ni poco, 4 casi como deseo y 5 tanto como deseo.

La dimensión confidencial, representa la opinión del cuidador sobre la posibilidad de contar con la comprensión y ayuda de personas al comunicar problemas íntimos, situaciones conflictivas o de otra índole; la dimensión afectiva evalúa el contar con personas que demuestren amor, cariño y empatía. Los puntos de corte del instrumento es de 33 puntos o más y se considera como apoyo social normal y con 32 puntos o menos apoyo social escaso(17).

Para el presente estudio las cinco posibles opciones de respuestas de la escala de Likert que evalúan el apoyo percibido, se agruparon en tres posibilidades, las opciones Mucho menos de lo que deseo y Menos de lo que deseo se agruparon en una y se denominó "Escasa ayuda", la opción "Ni mucho ni poco" quedó igual y las opciones Casi como deseo y Tanto como deseo se agruparon en una columna que se denominó "Mucha ayuda". Para el presente estudio se encontró un alfa de Cronbach de 0,876.

El almacenamiento y revisión de los datos obtenidos, se realizó en el programa de Microsoft Excel 2010, se interpretaron en el paquete estadístico SPSS (Statistical Package for the Social Sciences) versión 22.0, utilizando estadística descriptiva y de asociación para las variables cuantitativas. Se generaron análisis estadísticos utilizando porcentajes, tablas y gráficas para el análisis descriptivo de la información sobre las características sociodemográficas y del apoyo social percibido por los cuidadores familiares principales de niños con cáncer. Para estimar la asociación entre la percepción del apoyo social de los participantes con las condiciones sociodemográficas y de relación de cuidado, se calcularon Odds Ratio (OR), con intervalos de confianza del 95\%.

\section{Consideraciones éticas}

Se tuvo en cuenta los aspectos éticos y legales de acuerdo con los principios establecidos en la declaración de Helsinki y el artículo 10 de la Resolución 008430 de 1993. Este Estudio se clasificó de riesgo mínimo para los participantes, la incorporación a la investigación, fue realizada de manera voluntaria, luego de una explicación precisa sobre los alcances y objetivos de la misma. Se obtuvo el aval y el permiso correspondiente de las dos instituciones de salud donde se desarrolló el estudio. Los cuidadores que aceptaron participar firmaron el consentimiento informado, les fue entregado los instrumentos para su diligenciamiento con el acompañamiento de una de las investigadoras.

\section{Resultados}

De los cuidadores familiares principales de niños con cáncer que participaron en el estudio el 77,8\% (98) eran mujeres, en edades entre los 18 a 35 años el $47,7 \%$ (60) y el $45,2 \%$ (57) entre los 36 a 59 años. 
Con estudios completos e incompletos de primaria el $17,5 \%$ (22), de bachillerato el 53,2\% (67) y de formación superior el 27,7\% (35). El 65,1\% (82), tienen pareja estable y el 33,3\% (42) son solteros o separados. El 48,4\% (61) de los cuidadores se dedica a las actividades del hogar y el 49,2\% (62) trabajan de manera independiente o son empleados.

En cuanto a la relación que los une con la persona cuidada se encontró que en su mayoría son hijos, cuidadores principalmente desde el momento desde su diagnóstico, no se destaca en el grupo de estudio el ser único cuidador y tienen una experiencia menor a los 19 meses como cuidadores, esta labor le ocupa las 24 horas del día (Tabla 1).

\section{Apoyo social percibido por los cuidadores familiares principales de niños con cáncer}

Los cuidadores familiares principales de niños con cáncer consideraron que el apoyo social total recibido de acuerdo a los puntos de corte de la escala, fue percibido como normal en el 61,1\% (77) y escaso en el 38,9\% (49). En cuanto al apoyo social recibido de tipo confidencial el 75,4\% (95) de los cuidadores consideran que fue escaso, así como el $23,0 \%$ (29) el tipo de apoyo social afectivo (Tabla 2).

Tabla 1. Relación de cuidado de los cuidadores familiares principales de niños con cáncer. Cartagena, 2018

\begin{tabular}{|c|c|c|c|}
\hline Ítems & Características & $\mathbf{N}$ & $\%$ \\
\hline \multirow[t]{5}{*}{ Relación con la persona cuidada } & Hijo(a) & 84 & 66,7 \\
\hline & Nieto(a) & 18 & 14,3 \\
\hline & Sobrino(a) & 14 & 11,1 \\
\hline & Hermano(a) & 7 & 5,6 \\
\hline & Primo(a) & 3 & 2,3 \\
\hline \multirow[t]{2}{*}{ Cuida a la persona desde su diagnóstico } & $\mathrm{Si}$ & 119 & 94,4 \\
\hline & No & 7 & 5,6 \\
\hline \multirow[t]{2}{*}{ Único cuidador } & $\mathrm{Si}$ & 54 & 42,9 \\
\hline & No & 72 & 57,1 \\
\hline \multirow[t]{3}{*}{ Tiempo como cuidador } & 13 a 18 meses & 55 & 43,7 \\
\hline & 19 a 36 meses & 40 & 31,7 \\
\hline & Más de 37 meses & 31 & 24,6 \\
\hline \multirow{5}{*}{ Número de horas de cuidado } & Menos de 6 horas & 12 & 9,5 \\
\hline & 7 a 12 horas & 41 & 32,5 \\
\hline & 13 a 23 horas & 5 & 4,0 \\
\hline & 24 horas & 68 & 54 \\
\hline & Total & 126 & 100 \\
\hline
\end{tabular}

Fuente: Datos del estudio.

De los ítems que evalúan la percepción de apoyo confidencial, se encontró que "recibo visitas de los amigos y familiares" el 37,3\% (47) de los cuidadores respondieron que ni muchas ni pocas veces. A la pregunta "cuento con personas que se preocupan de lo que me sucede" el $72,2 \%$ (91) expresaron que recibieron mucha ayuda, igual respuesta se obtuvo del 50,8\% (64) a la pregunta "tengo la posibilidad de hablar con alguien de mis problemas en el trabajo o en la casa", igual respuesta se obtuvo del 61,9\% (78) al ítem "recibo consejos útiles cuando me ocurre algún acontecimiento importante en mi vida" lo mismo que para el $42,1 \%$ (53) ante la pregunta "tengo la posibilidad de hablar con alguien de los problemas personales y familiares". Al item "tengo la posibilidad de hablar con alguien de los problemas económicos" el 52,3\% (66) respondió que recibe poca ayuda ante esta situación (Tabla 3 ).
En cuanto al apoyo afectivo el 34,1\% (43) de los participantes respondió que recibe escasa ayuda a la pregunta "recibo ayuda en asuntos relacionados con mi casa", el 32,5\% (41) al item "recibo elogios y reconocimientos cuando hago bien mi trabajo" y el $54,8 \%$ (69) "recibo invitaciones para distraerme y salir con otras personas" (Tabla 3).

Tabla 2. Tipos de apoyo social percibidos por los cuidadores familiares principales de niños con cáncer

\begin{tabular}{llcc}
\hline \multicolumn{1}{c}{ Tipo de apoyo } & \multicolumn{1}{c}{ Nivel y puntaje } & $\mathbf{N}$ & $\mathbf{\%}$ \\
\hline Confidencial & Normal $\geq 19$ puntos & 31 & 24,6 \\
& Escaso $\leq 18$ puntos & 95 & 75,4 \\
\hline Afectivo & Normal $\geq 16$ puntos & 97 & 77 \\
& Escaso $\leq 15$ puntos & 29 & 23 \\
\hline \multirow{2}{*}{ Total } & Normal $\geq 33$ puntos & 77 & 61,1 \\
& Escaso $\leq 32$ puntos & 49 & 38,9 \\
& Total & 126 & 100
\end{tabular}


Tabla 3. Dimensiones de apoyo percibidos por los cuidadores familiares principales de niños con cáncer

\begin{tabular}{|c|c|c|c|c|c|c|c|}
\hline $\begin{array}{l}\text { Dimen- } \\
\text { siones }\end{array}$ & Items & $\begin{array}{l}\text { Escasa } \\
\text { ayuda }\end{array}$ & $\%$ & $\begin{array}{c}\mathrm{Ni} \\
\text { mucho ni } \\
\text { poco }\end{array}$ & $\%$ & $\begin{array}{l}\text { Mucha } \\
\text { ayuda }\end{array}$ & $\%$ \\
\hline \multirow{6}{*}{ 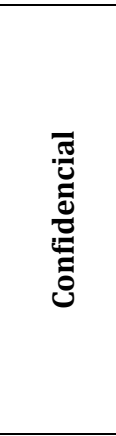 } & 1. Recibo visitas de mis amigos y familiares & 43 & 34,1 & 47 & 37,3 & 36 & 28,6 \\
\hline & $\begin{array}{l}\text { 4. Cuento con personas que se preocupan de lo } \\
\text { que me sucede }\end{array}$ & 8 & 6,4 & 27 & 21,4 & 91 & 72,2 \\
\hline & $\begin{array}{l}\text { 6. Tengo la posibilidad de hablar con alguien de } \\
\text { mis problemas en el trabajo o en la casa }\end{array}$ & 24 & 19 & 38 & 30,2 & 64 & 50,8 \\
\hline & $\begin{array}{l}\text { 7. Tengo la posibilidad de hablar con alguien mis } \\
\text { problemas personales y familiares }\end{array}$ & 33 & 26,2 & 40 & 31,7 & 53 & 42,1 \\
\hline & $\begin{array}{l}\text { 8. Tengo la posibilidad de hablar con alguien de } \\
\text { mis problemas económicos }\end{array}$ & 66 & 52,3 & 21 & 16,7 & 39 & 31 \\
\hline & $\begin{array}{l}\text { 10. Recibo consejos útiles cuando me ocurre } \\
\text { algún acontecimiento importante en mi vida }\end{array}$ & 11 & 8,7 & 37 & 29,4 & 78 & 61,9 \\
\hline \multirow{5}{*}{ 莺 } & $\begin{array}{l}\text { 2. Recibo ayuda en asuntos relacionados con mi } \\
\text { casa }\end{array}$ & 43 & 34,1 & 31 & 24,6 & 52 & 41,3 \\
\hline & $\begin{array}{l}\text { 3. Recibo elogios y reconocimientos cuando hago } \\
\text { bien mi trabajo }\end{array}$ & 41 & 32,5 & 25 & 19,9 & 60 & 47,6 \\
\hline & 5. Recibo amor y afecto & 7 & 5,6 & 10 & 7,9 & 109 & 86,5 \\
\hline & $\begin{array}{l}\text { 9. Recibo invitaciones para distraerme y salir } \\
\text { con otras personas }\end{array}$ & 69 & 54,8 & 26 & 20,6 & 31 & 24,6 \\
\hline & $\begin{array}{l}\text { 11. Recibo ayuda cuando estoy enfermo en la } \\
\text { cama }\end{array}$ & 40 & 31,7 & 24 & 19,1 & 62 & 49,2 \\
\hline
\end{tabular}

En la regresión logística realizada utilizando el Odds ratio $(O R)$ entre las variables sociodemográficas del cuidador familiar y de relación de cuidado entre el cuidador y el niño cuidado, ninguna de las variables presentó asociación significativa con el apoyo social (Tabla 4).

Tabla 4. Relación entre características de los cuidadores y el apoyo social requerido por los cuidadores familiares principales de niños con cáncer

\begin{tabular}{|c|c|c|c|c|}
\hline \multirow{2}{*}{ Variables } & \multirow{2}{*}{$\begin{array}{c}P \\
\text { value }\end{array}$} & \multirow{2}{*}{ OR } & \multicolumn{2}{|c|}{ OR I.C. 95\% } \\
\hline & & & Inferior & Superior \\
\hline Ser mujer & 0,795 & 0,868 & 0,297 & 2,534 \\
\hline $\begin{array}{l}\text { Cuidador de } 36 \text { años } \\
\text { y mas }\end{array}$ & 0,620 & 0,828 & 0,393 & 1,745 \\
\hline Sabe leer y escribir & 0,222 & 0,244 & 0,025 & 2,355 \\
\hline $\begin{array}{l}\text { Contar con } \\
\text { bachillerato en } \\
\text { adelante }\end{array}$ & 0,810 & 1,109 & 0,477 & 2,578 \\
\hline Tener pareja & 0,422 & 0,715 & 0,315 & 1,622 \\
\hline Tener un ingreso & 0,551 & 0,763 & 0,313 & 1,857 \\
\hline $\begin{array}{l}\text { Cuida a la persona } \\
\text { desde el inicio de su } \\
\text { dependencia }\end{array}$ & 0,576 & 0,600 & 0,100 & 3,594 \\
\hline $\begin{array}{l}\text { De } 19 \text { meses en } \\
\text { adelante como } \\
\text { cuidador }\end{array}$ & 0,825 & 0,919 & 0,436 & 1,937 \\
\hline $\begin{array}{l}\text { De } 13 \text { horas diarias } \\
\text { en adelante }\end{array}$ & 0,587 & 1,329 & 0,476 & 3,711 \\
\hline Ser único cuidador & 0,697 & 0,808 & 0,277 & 2,362 \\
\hline
\end{tabular}

\section{Discusión}

El apoyo social total percibido por los cuidadores familiares principales de niños con cáncer fue en su mayoría normal (61,1\%), sobre el tema se han realizado diferentes estudios como el de Melguizo et al.(18) quienes analizaron la influencia del apoyo social sobre el estrés en padres de niños con cáncer, destacando la importancia del apoyo recibido por la pareja en la reducción del estrés; al evaluar el apoyo social en cuidadores de pacientes dependientes(19), se encontró que éstos percibieron un bajo apoyo social $(36,1 \%)$ y una regular-mala calidad de vida relacionada con ansiedad y depresión.

Así mismo, en el estudio realizado a cuidadores de personas en tratamiento contra el cáncer, se reportó una percepción media y baja de soporte social en el $69,0 \%$ de los participantes(20). La necesidad de percibir el apoyo social por los cuidadores es de gran importancia para disminuir los efectos de tipo emocional que ocasiona desempeñar su labor, como lo demuestran los resultados de los estudios antes mencionados.

Ser cuidador familiar de un paciente con enfermedad crónica y en particular de niños con cáncer, se considera una situación desgastante por el impacto que genera en la familia el tratamiento, su 
adherencia y la pérdida de la vida social del niño, situación que exigen se destine la mayor parte de las horas del día a su cuidado (21).

Contar con apoyo social ya sea familiar o de personas cercanas es importante, porque proporciona fortaleza para afrontar la incertidumbre y ansiedad que con frecuencia están presentes, aun cuando la enfermedad se haya controlado(21). Para enfermería es necesario estimular en la familia la búsqueda de apoyo social a los cuidadores a fin de fortalecer su capacidad de afrontar la crisis familiar.

En el presente estudio se evidencia que los cuidadores familiares principales de niños, son generalmente sus padres, en su mayoría mujeres en la condición de madre $(66,7 \%)$, o abuela $(14,3 \%)$, similar a los hallazgos de otros estudios ${ }^{(14,21,22)}$ que confirman el rol social asignado a la mujer del cuidado familiar, responsabilidad que se atribuye a sus cualidades naturales para brindar cuidado, para Navarro y Carbonell(23) el cuidado informal se ha feminizado.

Los cuidadores del estudio presentaron edades entre los 18 y 59 años, similar a otros estudios(14,21), población que se encuentra en edad económicamente productiva y/o se dedican además a las actividades del hogar(20,21). El atender actividades laborales, del hogar y cuidar del niño con enfermedad oncológica es agotador y limitante, por cuanto impide atender las necesidades personales, haciendo evidente la necesidad de un mayor apoyo social $(7,12)$.

Al igual que en otros estudios asociados con cuidadores de niños con cáncer(14,21), los participantes de esta investigación cuentan principalmente con una formación académica de primaria y bachillerato completo o incompleto. Es importante que el personal de salud considere el nivel educativo de los cuidadores, de manera que pueda impartir orientaciones de cuidado claras y precisas para que adquieran una mayor habilidad de cuidado. Al respecto Castillo y Gamboa(24) y Tintaya(25), consideran que la educación facilita adquirir destrezas, resolver efectivamente problemas y desarrollar la personalidad.

El 66,1\% de los cuidadores cuenta con el apoyo de su pareja (casados, o en unión libre), dato que coincide con lo reportado en otras investigaciones ${ }^{(9,20-22) \text {, }}$ donde la pareja constituye un elemento de soporte.
Los cuidadores del estudio están dedicados a asistir y velar por el niño desde el momento que se le diagnosticó la enfermedad, dato similar al de Carrillo et al.(14). Esta condición le permite tener continuidad en el desarrollo del cuidado y por tanto, mejores conocimientos sobre los mismos, sin embargo, también mayor cansancio y necesidad de mayor apoyo social.

El cuidador familiar principal es el responsable de atender diariamente las necesidades del niño con cáncer, lo cual implica redistribuir sus actividades cumplirlas bajo una elevada carga de estrés, que se acentúa, si es el único cuidador(26-28). En el presente estudio un porcentaje significativo de los participantes son únicos cuidadores, similar a lo reportado en otras investigaciones en que más del $64 \%$ tienen esta condición(14,21,22).

Con relación al tiempo que llevan en ésta labor la mayoría tienen más de 19 meses, datos contrarios a lo encontrado por Carrillo et al.,(14,21) donde más del $50 \%$ de los participantes manifestaron tener una experiencia menor como cuidadores.

En esta investigación, los participantes aseguran dedicar 24 horas del día para el cuidado del menor las, similar a los estudios de Carrillo et al., (14,21) donde más del $70 \%$ de los cuidadores de niños con cáncer son de dedicación completa, tarea que a largo plazo conlleva a que el cansancio y el estrés se manifiesten, así como lo expresan Valencia et al. ${ }^{(29)} \mathrm{y}$ Carrillo et al. (21).

Por otra parte, los participantes perciben un escaso apoyo confidencial, similar al estudio de Flores y Seguel(12), donde el 98,5\% así lo reportan. El cuidador familiar principal de un niño con cáncer requiere del apoyo de familiares y amigos, ser escuchados y comprendidos, compartir con personas con las cuales pueda hablar sobre sus inquietudes y problemas personales y les ayuden a afrontar las situaciones difíciles propias de la enfermedad. Un porcentaje apreciable de los participantes lo percibió como escaso, resultados que concuerdan con Flores y Seguel(12) donde el 49,3\% de los cuidadores tuvieron una percepción similar.

Los cuidadores necesitan compartir sus sentimientos y sentirse valorados por las personas que lo rodean, de lo contrario, aumenta la carga emocional, generando actitudes y reacciones perturbadoras que provocan inestabilidad emocional. El apoyo social se 
constituye en un factor de protección para el cuidador familiar $(30,31)$.

De acuerdo a los resultados obtenidos en la regresión logística $O R$, no se presentó ninguna asociación significativa entre las variables sociodemográficas del cuidador familiar y de relación de cuidado entre el cuidador y el niño cuidado con el apoyo social percibido, contrario al estudio de Romero-Guevara et al.(32) quienes en su estudio encontraron diferencias estadísticamente significativas entre la escolaridad y el estrato socioeconómico con el soporte social. A su vez, Nightingale et al.(33) reportan, que los cuidadores consideran que a mayor carga de cuidado, disminuye la calidad de vida, y la percepción del apoyo social recibido se asocia con una menor carga de cuidado.

Asimismo, Romero-Guevara et al.(32) y Nightingale et al.(33), consideran que las intervenciones de enfermería deben orientarse a que los cuidadores identifiquen las fuentes de apoyo y a conocer cuándo y cómo buscarlas.

En relación a la posibilidad de que los cuidadores experimenten sobrecarga, Blanco et al.(34) concluyeron que el no tener un empleo fuera del hogar es un factor de riesgo, mientras que tener mayor edad y contar con suficiente apoyo social, es un factor protector. Por ello, se recomienda que los estudios de intervención se orienten a la implementación de programas para que los cuidadores identifiquen sus redes de apoyo y disminuyan los riesgos de la carga de cuidado ${ }^{(30,32) .}$

En la literatura se expresa que el apoyo social que recibe el cuidador es fundamental para enfrentar de manera efectiva las múltiples situaciones a la que está expuesto(7,12), pero en los resultados de este estudio no se presentó asociación significativa entre las características de los cuidadores y el apoyo social percibido. De las variables que se cruzaron estuvo el ser mujer (participaron 77,8\%) y saber leer $\mathrm{y}$ escribir (estudios hasta de bachillerato completo en el 70,7\%). Estos resultados confirman el rol social asignado a la mujer por sus cualidades naturales, $(7,14,21,22)$.

\section{Conclusiones}

Los resultados obtenidos en este estudio confirman que las mujeres son quienes asumen principalmente el rol de cuidadores, sin embargo, en esta investigación se ha encontrado que uno de cada cinco cuidadores de niños con cáncer, su cuidador es un hombre.

En el presente estudio no se encontró asociación entre las características sociodemográficas de los cuidadores familiares de niños con cáncer y el tipo de apoyo social (confidencial y afectivo) recibido. Resultado llamativo porque los participantes del estudio perciben como escaso, en un porcentaje apreciable, el apoyo social percibido.

Asumir el rol de cuidador a menudo se convierte en una situación de soledad y cansancio, llena de emociones difíciles de comprender, donde el deja de lado sus necesidades personales.

Se recomienda que el personal de enfermería realice estudios de intervención con los cuidadores y su familia, orientados a desarrollar valores en la convivencia, y de esta forma permitir la realización y reconocimiento de las actividades de apoyo social.

\section{Agradecimientos}

Por su participación en el estudio, a las cuidadoras de niños con cáncer, a las enfermeras Kelly Johanna Álvarez Banquett y Yilanis Paola Peroza Bahoque y a las instituciones de salud que permitieron acceder a su información.

\section{Referencias}

1. Organización de las Naciones Unidas. El cáncer infantil aumentó un 13\% en dos décadas, revela nuevo estudio de la OMS. Objetivos del Desarrollo Sostenible. 2017. p. 1.

2. Organización Mundial de la Salud. El cáncer infantil. 2018. p. 1 .

3. Instituto Nacional de Cancerología-ESE. Boletín Epidemiológico. 9. 2016. p. 1-18.

4. Alcaldia Mayor de Cartagena de Indias. Comportamiento de cáncer infantil (CI) año 2015, SIVIGILA Cartagena. 2015. p. $1-9$.

5. NIH NATIONAL CANCER INSTITUTE. Coping with cancer, Changes for the Family. NIH NATIONAL CANCER INSTITUTE. 2018. p. 1-4.

6. Montero Pardo X, Jurado Cárdenas S, Mendez Venegas J. Variables que predicen la aparición de sobrecarga en cuidadores primarios informales de niños con cáncer. PSICOONCOLOGIA. 2015;12(1):67-86.

7. Fernández Lao I, Del Pino Berenguer M, Silvano Arranz A. Percepción del cuidado por parte del cuidador familiar. Index enferm [Internet]. 2013;22(1/2):12-20. Available from:

http://scielo.isciii.es/scielo.php?script=sci_arttext\&pid=S11 32-12962013000100003\&lng=es\&nrm=iso\&tlng=es

8. Pinzón EA, Carrillo GM. Carga del cuidado y calidad de vida en cuidadores familiares de personas con enfermedad respiratoria crónica. Rev Fac Nac Salud Pública. 2016;34(2):193-201. 
9. Pozo Muñoz C, Bretones Nieto B, Martos Méndez MJ, Alonso Morillejo E, Cid Carrique N. Repercusiones psicosociales del cáncer infantil: Apoyo social y salud en familias afectadas. Rev Latinoam Psicol. 2015;47(2):93-101.

10. Torres-Pinto $\mathrm{X}$, Carreño-Moreno S, Chaparro-Díaz L. Factores que influencian la habilidad y sobrecarga del cuidador familiar del enfermo crónico. Rev Univ Ind Santander Salud [Internet]. 2017;49(2):330-8. Available from: http://dx.doi.org/10.18273/revsal.v49n2-2017006

11. Achury Saldaña DM, Restrepo Sánchez A, Torres Castro NM, Buitrago Mora AL, Neira Beltrán NX, Devia Florez P. Competencia de los cuidadores familiares para cuidar a los pacientes con falla cardíaca. Rev Cuid [Internet]. 2017;8(3):1721-32. Available from: http://www.revistacuidarte.org/index.php/cuidarte/article /download/367/759

12. Flores González E, Seguel Palma F. Functional social support in family caregivers of elderly adults with severe dependence. Invest educ enferm. 2016;34(1):67-73.

13. Ostertag Antezana FN. Soporte social en el cuidador primario de una persona con Alzheimer. Ajayu. 2014;12(1):79-99.

14. Carrillo GM, Sánchez-Herrera B, Barrera-Ortiz L. Habilidad de cuidado de cuidadores familiares de niños con cáncer. Rev salud pública. 2015;17(3):394-403.

15. Vergara Mercado M, Barrera Ortiz L. Confiabilidad del instrumento "habilidad de cuidado de cuidadores de pacientes con enfermedades crónicas". Av enferm. 2013;31(2):12-20.

16. Suarez Cuba MÁ. Identificación y utilidad de las herramientas para evaluar el apoyo social al paciente y al cuidador informal. Rev Med La Paz. 2011;17(1):60-7.

17. Azcárate-García E, Valle-Matildes U, Villaseñor-Hidalgo R, Gómez-Pérez AI. Apoyo social a mujeres con cáncer de mama en una unidad de medicina familiar de la Ciudad de México. ATEN FAM. 2017;24(4):169-72.

18. Melguizo-Garín A, Martos-Méndez MJ, Hombrados-Mendieta I. Influencia del apoyo social sobre el estrés y la satisfacción vital en padres de niños con cáncer desde una perspectiva multidimensional. Psicooncología. 2019;16(1):25-42.

19. Santos García CN, Ponce Troncoso A, Vieytes Oliva N, Beltrán Martínez A. ¿Cómo son las cuidadoras de pacientes dependientes de un centro de atención primaria? In: Salud, alimentación y sexualidad en el ciclo vital Volumen I [Internet]. 2016:257-61. Available from: http://formacionasunivep.com/IIIcongresosalud/document s/Libro Salud, alimentacion y sexualidad en el ciclo vital Volumen I.pdf\#page $=168$

20. Puerto Pedraza HM. Soporte Social Percibido En Cuidadores Familiares De Personas En Tratamiento Contra El Cáncer. Rev Cuid [Internet]. 2017;8(1):1407-22. Available from: http://www.scielo.org.co/pdf/cuid/v8n1/2216-0973-cuid8-01-01407.pdf

21. Carrillo GM, Barrera Ortiz L, Sánchez Herrera B, Carreño SP, Chaparro Díaz L. Efecto del programa de habilidad de cuidado para cuidadores familiares de niños con cáncer. Rev Colomb Cancerol. 2014;18(1):18-26.
22. Puerto Pedraza HM, Carrillo González GM. Calidad de vida y soporte social en los cuidadores familiares de personas en tratamiento contra el cancer. Rev Univ Ind Santander Salud. 2015;47(2):125-136

23. Navarro Pérez JJ, Carbonell Marqués Á. Sobrecarga familiar, apoyo social y salud comunitaria en cuidadores de personas con trastorno mental grave. Rev Esc Enferm USP. 2018;52(e03351):1-8.

24. Castillo Sánchez M, Gamboa Araya R. Desafíos de la educación en la sociedad actual. Dialogos Educ. 2012;12:5569.

25. Tintaya Condori P. Enseñanza y desarrollo personal. Revista de Psicología. 2016(16);75-86. Disponible en: http://www.scielo.org.bo/scielo.php?script=sci_arttext \&pid=S2223-30322016000200005

26. Giraldo Montoya DI, Zuluaga Machado S, Uribe Gómez V. Sobrecarga en los cuidadores principales de pacientes con dependencia permanente en el ámbito ambulatorio. Med UPB. 96. https://doi.org/10.18566/medupb.v37n2.a02

27. Pérez Rodríguez M, Álvarez Gómez T, Martínez Badaló E, Valdivia Cañizares S, Borroto Carpio I, Pedraza Núñez H. El síndrome del cuidador en cuidadores principales de ancianos con demencia Alzhéimer. Gac Médica Espirituana. 2017;19(1):38-50.

28. Sánchez Martínez RT, Molina Cardona EM, Gómez-Ortega OR. Intervenciones de enfermería para disminuir la sobrecarga en cuidadores: un estudio piloto. Rev Cuid. 2016;7(1):1171-84.

29. Valencia MC, Meza-Osnaya G, Pérez-Cruz I, Cortes-Campero N, Hernández-Ovalle J, Hernández-Paredes $\mathrm{P}$, et al. Factores que intervienen en la sobrecarga del cuidador primario del paciente con cáncer. Rev Calid Asist. 2017;32(4):221-5.

30. Barrera Ortiz L, Campos MS, Gallardo-Solarte K, CoralIbarra RDC, Hernández-Bustos A. Soporte social percibido por las personas con enfermedad crónica y sus cuidadores familiares en cinco macro regiones geográficas de Colombia. Univ y Salud. 2016;18(1):102-12.

31. Maluche Sánchez A, Velásquez VF. Efecto Programa De Apoyo Social: Percepción De Cuidadoras Familiares De Personas Mayores Afrocolombianas En Guapi, Cauca. Hacia prom salud. 2017;22(2):99-110.

32. Romero-Guevara SL, Correa BL, Camargo-Figuera FA. Soporte social percibido en cuidadores familiares de personas con enfermedad crónica. Univ y Salud. 2017;19(1):85-93.

33. Nightingale CL, Curbow BA, Wingard JR, Pereira DB, Carnaby GD. Burden, quality of life, and social support in caregivers of patients undergoing radiotherapy for head and neck cancer: A pilot study. Chronic Illn [Internet]. 2016;12(3):236-45. Available from: https://doi.org/10.1177/1742395316644305

34. Blanco V, Guisande MA, Sánchez MT, Otero P, López L, Vázquez FL. Síndrome de carga del cuidador y factores asociados en cuidadores familiares gallegos. Rev Esp Geriatr Gerontol [Internet]. 2019;54(1):19-26. Available from: https://doi.org/10.1016/j.regg.2018.03.005 